\title{
Spatial Approximation of Nondivergent Type Parabolic PDEs with Unbounded Coefficients Related to Finance
}

\author{
Fernando F. Gonçalves ${ }^{1,2}$ and Maria Rosário Grossinho ${ }^{1}$ \\ ${ }^{1}$ CEMAPRE \& Departmento de Matemática, ISEG, Universidade de Lisboa, Rua do Quelhas 6, 1200-781 Lisboa, Portugal \\ ${ }^{2}$ Universidade Europeia, Estrada da Correia 53, 1500-210 Lisboa, Portugal
}

Correspondence should be addressed to Fernando F. Gonçalves; fgoncalves@iseg.ulisboa.pt

Received 26 July 2013; Accepted 5 January 2014; Published 6 March 2014

Academic Editor: István Györi

Copyright (C) 2014 F. F. Gonçalves and M. R. Grossinho. This is an open access article distributed under the Creative Commons Attribution License, which permits unrestricted use, distribution, and reproduction in any medium, provided the original work is properly cited.

\begin{abstract}
We study the spatial discretisation of the Cauchy problem for a multidimensional linear parabolic PDE of second order, with nondivergent operator and unbounded time- and space-dependent coefficients. The equation free term and the initial data are also allowed to grow. Under a nondegeneracy assumption, we consider the PDE solvability in the framework of the variational approach and approximate in space the PDE problem's generalised solution, with the use of finite-difference methods. The rate of convergence is estimated.
\end{abstract}

\section{Introduction}

In this paper, we study the discretisation in space of the Cauchy problem

$$
\begin{gathered}
\frac{\partial u}{\partial t}=L u+f \quad \text { in }[0, T] \times \mathbb{R}^{d}, \\
u(0, x)=g(x) \quad \text { on } \mathbb{R}^{d},
\end{gathered}
$$

where $L$ is the second-order partial differential operator in the nondivergence form

$$
\begin{array}{r}
L(t, x)=a^{i j}(t, x) \frac{\partial^{2}}{\partial x^{i} \partial x^{j}}+b^{i}(t, x) \frac{\partial}{\partial x^{i}}+c(t, x), \\
i, j=1, \ldots, d,
\end{array}
$$

with real coefficients (written with the usual summation convention), $f$ and $g$ are given real-valued functions, and $T \in(0, \infty)$ is a constant. We assume that operator $\partial / \partial t-L$ is uniformly parabolic and allows the growth in the spatial variables of the first- and second-order coefficients in $L$ (linear and quadratic growth, resp.) and of the data $f$ and $g$ (polynomial growth).

Multidimensional partial differential equation (PDE) problems arise in Financial Mathematics and in Mathematical Physics. We are mainly motivated by the application to a large class of stochastic models in Financial Mathematics comprising the non-path-dependent options, with fixed exercise, written on multiple assets (basket options, exchange options, compound options, European options on future contracts and foreign-exchange, and others) and also to a particular type of path-dependent options: the Asian options (see, e.g., [1]).

Let us consider the stochastic modelling of a multiasset option of European type under the framework of a general version of Black-Scholes model, where the vector of asset appreciation rates and the volatility matrix are taken to be time- and space-dependent, and the riskless interest rate is a function of time. Owing to a Feynman-Kač type formula, pricing this option can be reduced to solving the Cauchy problem (with terminal condition) for the degenerate second-order linear parabolic PDE of nondivergent type, with null term and unbounded coefficients (see, e.g., [1]),

$$
\begin{array}{r}
\frac{\partial V}{\partial t}+\frac{1}{2} \sigma^{i j}(t, S) S^{i} S^{j} \frac{\partial^{2} V}{\partial S^{i} \partial S^{j}}+r(t) S^{i} \frac{\partial V}{\partial S^{i}}-r(t) V=0 \\
\text { in }[0, T] \times \mathbb{R}_{+}^{d}, \\
V(T, S)=\phi(S) \text { on } \mathbb{R}_{+}^{d},
\end{array}
$$


where $\mathbb{R}_{+}^{d} \equiv\left\{x \in \mathbb{R}^{d}: x^{i}>0, i=1, \ldots, d\right\}, V$ is the (unknown) option value, $S^{i}$ the price of the $i$ th underlying asset, $\left(\sigma^{i j}\right)$ the volatility matrix, $r$ the risk-free interest rate, and $\phi$ the pay-off function.

Therefore, as an alternative to approximating the option price with probabilistic numerical methods, we can approximate the solution of the corresponding PDE problem (3) with the use of nonprobabilistic techniques.

When problem (1) is considered in connection with option pricing, we see that the growth of the Black-Scholes PDE coefficients is appropriately matched. Also, the general case where the asset appreciation rate vector, the volatility matrix, and the risk-free interest rate are variable is covered. Finally, by imposing weak conditions on the initial data $g$, we will allow the financial derivative pay-off to be specified in a large class of functions. The free term $f$ is included to further improve generality.

In this paper, we study the approximation in space (for the time approximation, we refer to [2-4], where a general evolution equation problem of parabolic type is discretised) of the second-order parabolic problem (1), in the challenging case where the coefficients are unbounded (as well as the free data $f$ and $g$ ). The results are obtained under the strong assumption that the PDE does not degenerate but by imposing weak regularity assumptions. In order to facilitate the approach, we avoid any numerical methods' sophistication and make use of basic one-step finite-difference schemes. Also, an estimate for the rate of convergence of the discretised problem's generalised solution to the exact problem's generalised solution is given.

The numerical methods and possible approximation results are strongly linked to the theory on the solvability of the PDEs. We make use of the $L^{2}$ theory of solvability of linear PDEs in weighted Sobolev spaces. In particular, we consider the PDE solvability in a class of weighted Sobolev spaces used by O. G. Purtukhia (the references for Purtukhia's works can be found in [5]) for the treatment of linear stochastic partial differential equations (SPDEs) and further generalised by Gyöngy and Krylov (see [5]), the so-called well-weighted Sobolev spaces. By constructing discrete versions of these spaces, we set a suitable discretised framework and investigate the spatial approximation to the PDE generalised solution with the use of standard variational techniques.

We emphasize some points.

Firstly, we note that many PDE problems related to finance are Cauchy problems: initial-boundary value problems arise mostly after a localisation procedure for the purpose of obtaining implementable numerical schemes. Therefore, we do not find in many of these problems the complex domain geometries which are one important reason to favour other numerical methods (e.g., finite-element methods).

Also, although the finite-difference method for approximating PDEs is a well-developed area, and the theory could be considered reasonably complete since three decades ago, some important research is still currently pursued (see, e.g., the recent works [8-10]). (We refer to [6] for a brief summary of the method's history, and also for the references of the seminal works by R. Courant, K. O. Friedrichs, and H. Lewy, and further major contributions by many others. For the application of the finite-difference method to option pricing, we refer to the review paper [7] for the references of the original publications by M. Brennan and E. S. Schwartz and further major research.)

Secondly, we observe that the usual procedure for obtaining implementable numerical schemes for problem (1) is to localise it to a bounded domain in $[0, T] \times \mathbb{R}^{d}$ and then to approximate the localised problem (see, e.g., [11-13]; see also [14], where the approximation is pursued for more complex financial models but using the same localisation technique). In this case, there is no need to consider weighted functional spaces for the solvability and approximation study, as the PDE coefficients are bounded in the truncated domain.

An alternative procedure is to (semi)discretise problem (1) in $[0, T] \times Z_{h}^{d}$, with $Z_{h}^{d}$ being the $h$-grid on $\mathbb{R}^{d}$, and then localise the discretised problem to a bounded domain in $[0, T] \times Z_{h}^{d}$ by imposing a discrete artificial boundary condition (see, e.g., [15-17], where several types of initialvalue problems on unbounded domains are approximated; we refer to $[16,17]$ for the procedure discussion). Our study is meaningful in this latter case, as the coefficient unboundedness remains a problem that must be dealt with.

Finally, we remark that (i) the partial differential operators arising in finance are of nondivergent type and (ii) we do not assume the operator coefficients to be smooth enough to be possible to obtain an equivalent divergent operator. Therefore, although there are definite advantages in considering the operator in the divergent form when the approach is variational, this is not available for the present work.

We outline the paper. In Section 2, we establish some wellknown facts on the solvability of linear PDEs under a general framework and introduce the well-weighted Sobolev spaces. In Section 3, we discretise in space problem (1), with the use of a finite-difference scheme. We set a discrete framework and deduce the existence and uniqueness of the discretised problem's generalised solution. In Section 4, we investigate the approximation properties of the scheme and compute a rate of convergence. In Section 5, we make a few final comments.

\section{Preliminaries and Classical Results}

We establish some facts on the solvability of PDEs under a general framework.

Let $V$ be a reflexive separable Banach space embedded continuously and densely into a Hilbert space $H$ with inner product (,). Then $H^{*}$, the dual space of $H$, is also continuously and densely embedded into $V^{*}$, the dual of $V$. Let us use the notation $\langle$,$\rangle for the duality. Let H^{*}$ be identified with $H$ in the usual way, by the help of the inner product. Then we have the so-called normal triple $V \hookrightarrow H \equiv H^{*} \hookrightarrow V^{*}$, with continuous and dense embeddings. 
Let us consider the Cauchy problem for an evolution equation

$$
\frac{d u}{d t}=A(t) u+f(t) \quad \text { in }[0, T], u(0)=g,
$$

with $T \in(0, \infty)$ and where for every $t \in[0, T] A(t)$ is a linear operator from $V$ to $V^{*}, f(t) \in V^{*}$, and $g \in H$.

We assume that the operator $A(t)$ is continuous and impose a coercivity condition, as well as some regularity on the free data $f$ and $g$.

Assumption 1. There exist constants $\lambda>0, K, M$, and $N$ such that

(1) $\langle A(t) v, v\rangle+\lambda\|v\|_{V}^{2} \leq K\|v\|_{H}^{2}, \forall v \in V, \forall t \in[0, T]$;

(2) $\|A(t) v\|_{V^{*}} \leq M\|v\|_{V}, \forall v \in V, \forall t \in[0, T]$;

(3) $\int_{0}^{T}\|f(t)\|_{V^{*}}^{2} d t \leq N$ and $\|g\|_{H} \leq N$.

We define the generalised solution of problem (4).

Definition 2. One says that $u \in C([0, T] ; H)$ is a generalised solution of $(4)$ on $[0, T]$ if

(1) $u \in L^{2}([0, T] ; V)$;

(2) for every $t \in[0, T]$,

$$
(u(t), v)=(g, v)+\int_{0}^{t}\langle A(s) u(s), v\rangle d s+\int_{0}^{t}\langle f(s), v\rangle d s
$$

holds for all $v \in V$.

Notation 1. Let $W$ be a Banach space with norm \|\| . We denote by $C([0, T] ; W)$ the space of continuous $W$-valued functions on $[0, T]$ and by $L^{2}([0, T] ; W)$ the space of $W$ valued functions $w$ on $[0, T]$ such that $\|w\|_{L^{2}([0, T] ; W)}:=$ $\left(\int_{0}^{T}\|w(t)\|^{2} d t\right)^{1 / 2}<\infty$.

The following well-known result states that, under Assumption 1, problem (4) has a unique generalised solution (see, e.g., [5], where the result is stated for the more general case of a linear stochastic evolution equation problem and [18]).

Theorem 3. Under (1)-(3) in Assumption 1, problem (4) has a unique generalised solution on $[0, T]$. Moreover

$$
\begin{aligned}
\sup _{t \in[0, T]}\|u(t)\|_{H}^{2}+\int_{0}^{T}\|u(t)\|_{V}^{2} d t \\
\quad \leq N\left(\|g\|_{H}^{2}+\int_{0}^{T}\|f(t)\|_{V^{*}}^{2} d t\right),
\end{aligned}
$$

where $N$ is a constant.

Let us now consider the particular PDE problem

$$
u_{t}=L u+f \quad \text { in } Q, \quad u(0, x)=g(x) \quad \text { on } \mathbb{R}^{d},
$$

where $L$ is the second-order operator with real coefficients

$$
L(t, x)=a^{i j}(t, x) \frac{\partial^{2}}{\partial x^{i} \partial x^{j}}+b^{i}(t, x) \frac{\partial}{\partial x^{i}}+c(t, x),
$$

$Q=[0, T] \times \mathbb{R}^{d}$, with $T \in(0, \infty)$, and $f$ and $g$ are given functions. We allow the growth, in the spatial variables, of the coefficients $a^{i j}(t, x)$ and $b^{i}(t, x), i, j=1, \ldots, d$, and of the free data $f(t, x)$ and $g(x)$.

In order to set the framework for problem (7), we introduce a suitable class of weighted Sobolev spaces, the socalled well-weighted Sobolev spaces (we refer to [5] for a complete description of this class of spaces).

Let $U$ be a domain in $\mathbb{R}^{d}$, that is, an open subset of $\mathbb{R}^{d}$. Let $r>0, \rho>0$ be smooth functions in $U$ and $m \geq 0$ an integer. The weighted Sobolev space $W^{m, 2}(U ; r, \rho)$ is the Banach space of locally integrable functions $v: U \rightarrow \mathbb{R}$ such that for each multi-index $\alpha$, with $|\alpha| \leq m, D^{\alpha} v$ exists in the weak sense, and

$$
\|v\|_{W^{m, 2}(U ; r, \rho)}:=\left(\sum_{|\alpha| \leq m} \int_{U} r^{2}\left|\rho^{|\alpha|} D^{\alpha} v\right|^{2} d x\right)^{1 / 2}<\infty .
$$

Endowed with the inner product which generates the above norm

$$
(v, w)_{W^{m, 2}(U ; r, \rho)}:=\sum_{|\alpha| \leq m} \int_{U} r^{2} \rho^{2|\alpha|} D^{\alpha} v D^{\alpha} w d x,
$$

for all $v, w \in W^{m, 2}(U ; r, \rho), W^{m, 2}(U ; r, \rho)$ is a Hilbert space.

Remark 4. Setting the weight functions $r=\rho=1$, for all $x \in U$, we obtain the particular case of the Sobolev spaces $W^{m, 2}(U)$.

Notation 2. In the sequel, when $U=\mathbb{R}^{d}$ we drop the argument in the function space notation. For instance, we denote $W^{m, 2}\left(\mathbb{R}^{d} ; r, \rho\right)=: W^{m, 2}(r, \rho)$.

We make some assumptions on the behaviour of the weight functions $r$ and $\rho$ (see [5]).

Assumption 5. Let $m \geq 0$ be an integer and $r>0, \rho>0$ smooth functions on $\mathbb{R}^{d}$. There exists a constant $K$ such that

(1) $\left|D^{\alpha} \rho\right| \leq K \rho^{1-|\alpha|}$, for all multi-indexes $\alpha$ such that $|\alpha| \leq m-1$ if $m \geq 2$;

(2) $\left|D^{\alpha} r\right| \leq K\left(r / \rho^{|\alpha|}\right)$, for all multi-indexes $\alpha$ such that $|\alpha| \leq m$.

Remark 6. In (1) in Assumption 5, if $m<2$ nothing is required.

Example 7. The following functions (taken from [5], citing O. G. Purtukhia) satisfy Assumption 5:

(1) $r(x)=\left(1+|x|^{2}\right)^{\beta}, \beta \in \mathbb{R} ; \rho(x)=\left(1+|x|^{2}\right)^{\gamma}, \gamma \leq 1 / 2$;

(2) $r(x)=\exp \left( \pm\left(1+|x|^{2}\right)^{\beta}\right), 0 \leq \beta \leq 1 / 2 ; \rho(x)=(1+$ $\left.|x|^{2}\right)^{\gamma}, \gamma \leq 1 / 2-\beta$ 
(3) $r(x)=\left(1+|x|^{2}\right)^{\beta}, \beta \in \mathbb{R} ; \rho(x)=\ln ^{\gamma}\left(2+|x|^{2}\right), \gamma \in \mathbb{R}$;

(4) $r(x)=\left(1+|x|^{2}\right)^{\beta} \ln ^{\mu}\left(2+|x|^{2}\right), \beta \geq 0, \mu \geq 0 ; \rho(x)=$ $\left(1+|x|^{2}\right)^{\gamma}, \gamma \leq 1 / 2$

(5) $r(x)=\left(1+|x|^{2}\right)^{\beta} \ln ^{\mu}\left(2+|x|^{2}\right), \beta \geq 0, \mu \geq 0 ; \rho(x)=$ $\ln ^{\gamma}\left(2+|x|^{2}\right), \gamma \geq 0$

(6) $\rho(x)=\exp \left(-\left(1+|x|^{2}\right)^{\gamma}\right), \gamma \geq 0$; each weight function $r(x)$ in examples (1)-(5).

Now, we switch our point of view and consider the functions $w: Q \rightarrow \mathbb{R}$ as mappings of $t$ into certain spaces of functions of $x$ we make precise below such that, for all $t \in[0, T], x \in \mathbb{R}^{d},(w(t))(x):=w(t, x)$.

We impose a coercivity condition and make some assumptions on the growth and regularity of the operator's coefficients and also on the regularity of the free data $f$ and $g$ (see [5], where the assumptions are made for the more general case of an SPDE problem).

Assumption 8. Let $r>0, \rho>0$ be smooth functions on $\mathbb{R}^{d}$ and $m \geq 0$ an integer.

(1) There exists a constant $\lambda>0$ such that $\sum_{i, j=1}^{d} a^{i j}(t, x) \xi^{i} \xi^{j} \geq \lambda \rho^{2}(x)|\xi|^{2}$, for all $t \geq 0$, $x \in \mathbb{R}^{d}$, and $\xi \in \mathbb{R}^{d}$.

(2) The coefficients of $L$ are measurable functions in $[0, T] \times \mathbb{R}^{d}$. The derivatives in $x$ of the coefficients $a^{i j}$ up to order $m \vee 1$ and of the coefficients $b^{i}$ and $c$ up to order $m$ exist for any $t \in[0, T]$. Moreover, there exists a constant $K$ such that

$$
\begin{gathered}
\left|D_{x}^{\alpha} a^{i j}\right| \leq K \rho^{2-|\alpha|} \quad \forall|\alpha| \leq m \vee 1, \\
\left|D_{x}^{\alpha} b^{i}\right| \leq K \rho^{1-|\alpha|}, \quad\left|D_{x}^{\alpha} c\right| \leq K \quad \forall|\alpha| \leq m,
\end{gathered}
$$

for all $t \in[0, T], x \in \mathbb{R}^{d}$, with $D_{x}^{\alpha}$ denoting the $|\alpha|$ th partial derivative operator with respect to $x$.

(3) Consider $f \in L^{2}\left([0, T] ; W^{m-1,2}(r, \rho)\right)$ and $g \in$ $W^{m, 2}(r, \rho)$.

Notation 3. In the above assumption, $p \vee q:=\max (p, q)$, with $p, q$ integers. Also, for $m=0$ we use the notation $W^{-1,2}(r, \rho):=\left(W^{1,2}(r, \rho)\right)^{*}$, where $\left(W^{1,2}(r, \rho)\right)^{*}$ is the dual of $W^{1,2}(r, \rho)$.

We define the generalised solution of problem (7).

Definition 9. One says that $u \in C\left([0, T] ; W^{0,2}(r, \rho)\right)$ is a generalised solution of $(7)$ on $[0, T]$ if

(1) $u \in L^{2}\left([0, T] ; W^{1,2}(r, \rho)\right)$;
(2) for every $t \in[0, T]$,

$$
\begin{aligned}
& (u(t), \varphi) \\
& =(g, \varphi)+\int_{0}^{t}\left\{-\left(a^{i j}(s) D_{x^{i}} u(s), D_{x^{j}} \varphi\right)\right. \\
& +\left(b^{i}(s) D_{x^{i}} u(s)-D_{x^{j}} a^{i j}(s) D_{x^{i}} u(s), \varphi\right) \\
& +(c(s) u(s), \varphi)+\langle f(s), \varphi\rangle\} d s
\end{aligned}
$$

holds for all $\varphi \in C_{0}^{\infty}$.

Notation 4. The notation (, ) in the above definition stands for the inner product in $W^{0,2}(r, \rho)$. Also, we denote $C_{0}^{\infty}:=$ $C_{0}^{\infty}\left(\mathbb{R}^{d}\right)$.

Remark 10. Note that as an alternative to the infinite differentiability of $\varphi$ in (2) it can be required that $\varphi \in W^{1,2}(r, \rho)$.

Finally, we state a result on the existence and uniqueness of the solution of problem (7). This result can be obtained from the general result in abstract spaces by using the suitable triple of spaces (see, e.g., [5], where the result is proved for the more general case of an SPDE problem).

Theorem 11. Under (1)-(2) in Assumption 5, with $m+1$ in place of $m$, with $m \geq 0$ being an integer, and (1)-(3) in Assumption 8, problem (7) admits a unique generalised solution $u$ on $[0, T]$. Moreover

$$
\begin{gathered}
u \in C\left([0, T] ; W^{m, 2}(r, \rho)\right) \cap L^{2}\left([0, T] ; W^{m+1,2}(r, \rho)\right), \\
\sup _{0 \leq t \leq T}\|u(t)\|_{W^{m, 2}(r, \rho)}^{2}+\int_{0}^{T}\|u(t)\|_{W^{m+1,2}(r, \rho)}^{2} d t \\
\leq N\left(\|g\|_{W^{m, 2}(r, \rho)}^{2}+\int_{0}^{T}\|f(t)\|_{W^{m-1,2}(r, \rho)}^{2} d t\right),
\end{gathered}
$$

with $N$ being a constant.

\section{The Discrete Framework}

We now proceed to the discretisation of problem (7) in the spatial variable. We set a suitable discrete framework with the use of a finite-difference scheme and, by showing that discretised problem can be cast into the general problem (4), we prove an existence and uniqueness result for the discretised problem's generalised solution.

We emphasize that this study mirrors the study of problem (7), in the sense that the framework we now set is a discrete version of the framework set for problem (7), and the techniques used for proving the existence and uniqueness results are the same for both problems.

Define the $h$-grid on $\mathbb{R}^{d}$, with $h \in(0,1]$,

$$
Z_{h}^{d}=\left\{x \in \mathbb{R}^{d}: x=h \sum_{i=1}^{d} e_{i} n_{i}, n_{i}=0, \pm 1, \pm 2, \ldots\right\},
$$


where $e_{i}$, for $i=1,2, \ldots, d$, is the unit vector in $\mathbb{R}^{d}$ whose $i$ th entry is 1 .

For every $x \in Z_{h}^{d}$, denote

$$
\begin{aligned}
& \partial_{i}^{+} u=\partial_{i}^{+} u(t, x)=h^{-1}\left(u\left(t, x+h e_{i}\right)-u(t, x)\right), \\
& \partial_{i}^{-} u=\partial_{i}^{-} u(t, x)=h^{-1}\left(u(t, x)-u\left(t, x-h e_{i}\right)\right)
\end{aligned}
$$

the forward and backward difference quotients in space, respectively. Define the discrete operator

$$
L_{h}(t, x)=a^{i j}(t, x) \partial_{j}^{-} \partial_{i}^{+}+b^{i}(t, x) \partial_{i}^{+}+c(t, x) .
$$

We consider the discrete problem

$$
u_{t}=L_{h} u+f_{h} \quad \text { in } Q(h), \quad u(0, x)=g_{h}(x) \quad \text { on } Z_{h}^{d} \text {, }
$$

where $Q(h)=[0, T] \times Z_{h}^{d}$, with $T \in(0, \infty)$, and $f_{h}$ and $g_{h}$ are functions such that $f_{h}: Q(h) \rightarrow \mathbb{R}$ and $g_{h}: Z_{h}^{d} \rightarrow \mathbb{R}$.

Consider functions $v: Z_{h}^{d} \rightarrow \mathbb{R}$. We introduce the space $l^{0,2}(r)$, the discrete version of the weighted Sobolev space $W^{0,2}(r, \rho)$,

$$
l^{0,2}(r):=\left\{v:\|v\|_{l^{0,2}(r)}<\infty\right\}
$$

where the norm $\|v\|_{l^{0,2}(r)}$ is given by

$$
\|v\|_{l^{0,2}(r)}=\left(\sum_{x \in Z_{h}^{d}} r^{2}(x)|v(x)|^{2} h^{d}\right)^{1 / 2} .
$$

We define, for any $v, w \in l^{0,2}(r)$, the inner product

$$
(v, w)_{l^{0,2}(r)}=\sum_{x \in Z_{h}^{d}} r^{2}(x) v(x) w(x) h^{d},
$$

which induces the norm.

The inner product space $l^{0,2}(r)$ has a good structure: it can be easily shown that it is complete, therefore a Hilbert space.

For functions $w: Z_{h}^{d} \rightarrow \mathbb{R}$, we introduce also the discrete version of the weighted Sobolev space $W^{1,2}(r, \rho)$, the space $l^{1,2}(r, \rho)$ defined by

$$
l^{1,2}(r, \rho)=\left\{w:\|w\|_{l^{1,2}(r, \rho)}<\infty\right\},
$$

with norm

$$
\|w\|_{l^{1,2}(r, \rho)}^{2}:=\|w\|_{l^{0,2}(r)}^{2}+\sum_{i=1}^{d}\left\|\rho \partial_{i}^{+} w\right\|_{l^{0,2}(r)}^{2} .
$$

We endow $l^{1,2}(r, \rho)$ with the inner product, inducing the norm,

$$
(w, z)_{l^{1,2}(r, \rho)}=(w, z)_{l^{0,2}(r)}+\sum_{i=1}^{d}\left(\rho \partial_{i}^{+} w, \rho \partial_{i}^{+} z\right)_{l^{0,2}(r)},
$$

for any functions $w, z \in l^{1,2}(r, \rho)$.
We want to show that the discrete framework we have set is a particular case of the general framework considered in Section 2.

It can be easily checked that $l^{1,2}(r, \rho)$ is a reflexive and separable Banach space, continuously and densely embedded into the Hilbert space $l^{0,2}(r)$.

As $l^{1,2}(r, \rho)$, endowed with the inner product $(,)_{l^{1,2}(r, \rho)}$, is clearly a Hilbert space therefore it is reflexive, and the proof for the separability is trivial. The continuity of the embedding follows immediately from $\|v\|_{l^{0,2}(r)} \leq\|v\|_{l^{1,2}(r, \rho)}$, for all $v \in$ $l^{1,2}(r, \rho)$. Finally, the denseness can be checked by noticing that, for an arbitrary function $w \in l^{0,2}(r)$, and $B$ a ball in $Z_{h}^{d}$, the function $z$ defined by

$$
z(x)= \begin{cases}w(x), & x \in B \\ 0, & \text { otherwise }\end{cases}
$$

belongs obviously to $l^{1,2}(r, \rho)$ and that, for any given $\varepsilon>0$, $\|w-z\|_{l^{0,2}(r)}<\varepsilon$ if the diameter of $B$ is chosen sufficiently large.

As in the previous section, we switch our viewpoint and consider the functions $z: Q(h) \rightarrow \mathbb{R}$ as mappings of $t$ into certain spaces of functions of $x$, defined by $(z(t))(x):=$ $z(t, x)$, for all $t \in[0, T]$ and for all $x \in Z_{h}^{d}$. For these functions, we consider the space $C\left([0, T] ; l^{0,2}(r)\right)$ of continuous $l^{0,2}(r)$ valued functions on $[0, T]$ and the spaces

$$
\begin{aligned}
L^{2} & \left([0, T] ; l^{m, 2}(r, \rho)\right) \\
& =\left\{z:[0, T] \longrightarrow l^{m, 2}(r, \rho): \int_{0}^{T}\|z(t)\|_{l^{m, 2}(r, \rho)}^{2} d t<\infty\right\},
\end{aligned}
$$

with $m=0,1$.

Notation 5. We identify $l^{0,2}(r, \rho)$ with $l^{0,2}(r)$.

Remark 12. Clearly, if $u \in C\left([0, T] ; l^{0,2}(r)\right)$ then $\sup _{t \in[0, T]}\|u(t)\|_{l^{0,2}(r)}<\infty$.

We make some assumptions on the regularity of the data $f_{h}$ and $g_{h}$ in (17).

Assumption 13. Let $r>0$ be a smooth function on $\mathbb{R}^{d}$. We assume

(1) $f_{h} \in L^{2}\left([0, T] ; l^{0,2}(r)\right)$;

(2) $g_{h} \in l^{0,2}(r)$.

Remark 14. In Assumption 13, (1) can be replaced with the weaker assumption $f_{h} \in L^{2}\left([0, T] ;\left(l^{1,2}(r, \rho)\right)^{*}\right)$, where $\left(l^{1,2}(r, \rho)\right)^{*}$ denotes the dual space of $l^{1,2}(r, \rho)$.

Remark 15. We note that $\left|\partial_{i}^{+} a^{i j}\right| \leq K \rho$ can be obtained from (2) in Assumption 8. In fact, by the mean-value theorem,

$$
\begin{aligned}
\left|\partial_{i}^{+} a^{i j}(t, x)\right| & =\left|h^{-1}\left(a^{i j}\left(t, x+h e_{i}\right)-a^{i j}(t, x)\right)\right| \\
& =\left|\frac{\partial}{\partial x^{i}} a^{i j}\left(t, x+\tau e_{i}\right)\right|,
\end{aligned}
$$


for some $\tau$ such that $0<\tau<h$. Thus $\left|\left(\partial / \partial x^{i}\right) a^{i j}\right| \leq K \rho$ implies $\left|\partial_{i}^{+} a^{i j}\right| \leq K \rho$.

We define the generalised solution of problem (17).

Definition 16. One says that $u \in C\left([0, T] ; l^{0,2}(r)\right) \cap L^{2}$ $\left([0, T] ; l^{1,2}(r, \rho)\right)$ is a generalised solution of (17) if, for every $t \in[0, T]$,

$$
\begin{aligned}
(u(t), \varphi)=\left(g_{h}, \varphi\right) & \\
+\int_{0}^{t}\{- & \left(a^{i j}(s) \partial_{i}^{+} u(s), \partial_{j}^{+} \varphi\right) \\
& +\left(b^{i}(s) \partial_{i}^{+} u(s)-\partial_{j}^{+} a^{i j}(s) \partial_{i}^{+} u(s), \varphi\right) \\
& \left.+(c(s) u(s), \varphi)+\left\langle f_{h}(s), \varphi\right\rangle\right\} d s
\end{aligned}
$$

holds for all $\varphi \in l^{1,2}(r, \rho)$.

Notation 6. In the above definition, $($,$) denotes the inner$ product in $l^{0,2}(r)$. We keep this convention for the remainder of present section.

Finally, we prove an existence and uniqueness result for the solution of the discrete problem (17). This result shows that the scheme is stable, that is, that, informally, the discrete problem's solution remains bounded independent of the space-step $h$. The result is obtained as a consequence of Theorem 3, remaining only to prove that, under the discrete framework we constructed, (1)-(2) in Assumption 1 hold.

Theorem 17. Under (1)-(2) in Assumption 8 and (1)-(2) in Assumption 13, problem (17) has a unique generalised solution $u$ in $[0, T]$. Moreover

$$
\begin{aligned}
& \sup _{0 \leq t \leq T}\|u(t)\|_{l^{0,2}(r)}^{2}+\int_{0}^{T}\|u(t)\|_{l^{1,2}(r, \rho)}^{2} d t \\
& \quad \leq N\left(\left\|g_{h}\right\|_{l^{0,2}(r)}^{2}+\int_{0}^{T}\left\|f_{h}(t)\right\|_{l^{0,2}(r)}^{2} d t\right),
\end{aligned}
$$

with $N$ a constant independent of $h$.

Proof. Let $L_{h}(s): l^{1,2}(r, \rho) \rightarrow\left(l^{1,2}(r, \rho)\right)^{*}$, for every $s \in$ $[0, T]$. We define

$$
\begin{aligned}
\left\langle L_{h}(s) \psi, \varphi\right\rangle:= & -\left(a^{i j}(s) \partial_{i}^{+} \psi, \partial_{j}^{+} \varphi\right) \\
& +\left(b^{i}(s) \partial_{i}^{+} \psi-\partial_{j}^{+} a^{i j}(s) \partial_{i}^{+} \psi, \varphi\right) \\
& +(c(s) \psi, \varphi),
\end{aligned}
$$

for all $s \in[0, T], \varphi, \psi \in l^{1,2}(r, \rho)$.

It suffices to prove that the following properties hold:

(1) $\exists K, \lambda>0$ constants: $\left\langle L_{h}(s) \psi, \psi\right\rangle+\lambda\|\psi\|_{l^{1,2}(r, \rho)} \leq$ $K\|\psi\|_{l^{0,2}(r)}$,

(2) $\exists K$ constant: $\left\langle L_{h}(s) \psi, \varphi\right\rangle \mid \leq K\|\psi\|_{l^{1,2}(r, \rho)} \cdot\|\varphi\|_{l^{1,2}(r, \rho)}$,

for all $s \in[0, T], \varphi, \psi \in l^{1,2}(r, \rho)$.
For the first property, owing to (1) and (2) in Assumption 8 , we have

$$
\begin{aligned}
\left\langle L_{h}(s) \psi, \psi\right\rangle= & -\sum_{i, j} \sum_{x} r^{2} a^{i j}(s) \partial_{i}^{+} \psi \partial_{j}^{+} \psi h^{d} \\
& +\sum_{i} \sum_{x} r^{2}\left(b^{i}(s)-\partial_{j}^{+} a^{i j}(s)\right) \partial_{i}^{+} \psi \psi h^{d} \\
& +\sum_{x} r^{2} c(s) \psi \psi h^{d} \\
\leq & -\lambda \sum_{i} \sum_{x} r^{2}\left|\rho \partial_{i}^{+} \psi\right|^{2} h^{d} \\
& +2 K \sum_{i} \sum_{x} r^{2} \rho\left|\partial_{i}^{+} \psi \psi\right| h^{d}+K \sum_{x} r^{2}|\psi|^{2} h^{d} \\
= & -\lambda \sum_{i}\left\|\rho \partial_{i}^{+} \psi\right\|_{l^{0,2}(r)}^{2} \\
& +2 K \sum_{i} \sum_{x} r^{2} \rho\left|\partial_{i}^{+} \psi \psi\right| h^{d}+K\|\psi\|_{l^{0,2}(r)}^{2}
\end{aligned}
$$

where the variable $x \in Z_{h}^{d}$ is omitted, $\sum_{x}$ denotes the summation over $Z_{h}^{d}$, and $\sum_{i}, \sum_{j}$ the summation over $\{1,2, \ldots, d\}$. We use Cauchy's inequality on the second term in estimate (30) and obtain

$$
\begin{aligned}
\left\langle L_{h}(s) \psi, \psi\right\rangle \leq & -\lambda \sum_{i}\left\|\rho \partial_{i}^{+} \psi\right\|_{l^{0,2}(r)}^{2}+\varepsilon K \sum_{i} \sum_{x} r^{2}\left|\rho \partial_{i}^{+} \psi\right|^{2} h^{d} \\
& +\frac{K}{\varepsilon} \sum_{i} \sum_{x} r^{2}|\psi|^{2} h^{d}+K\|\psi\|_{l^{0,2}(r)}^{2} \\
= & -\lambda \sum_{i}\left\|\rho \partial_{i}^{+} \psi\right\|_{l^{0,2}(r)}^{2}-\lambda\|\psi\|_{l^{0,2}(r)}^{2} \\
& +\varepsilon K \sum_{i}\left\|\rho \partial_{i}^{+} \psi\right\|_{l^{0,2}(r)}^{2} \\
& +\frac{K}{\varepsilon}\|\psi\|_{l^{0,2}(r)}^{2}+(K+\lambda)\|\psi\|_{l^{0,2}(r)}^{2} \\
\leq & -\lambda\|\psi\|_{l^{1,2}(r, \rho)}^{2}+K\|\psi\|_{l^{0,2}(r)}^{2}
\end{aligned}
$$

with $\lambda>0, K$ constants, by taking $\varepsilon$ sufficiently small. The first property is proved.

The second property follows from (2) in Assumption 8 and Cauchy-Schwarz inequality

$$
\begin{aligned}
\left|\left\langle L_{h}(s) \psi, \varphi\right\rangle\right|=\mid- & \sum_{i, j} \sum_{x} r^{2} a^{i j}(s) \partial_{i}^{+} \psi \partial_{j}^{+} \varphi h^{d} \\
& +\sum_{i} \sum_{x} r^{2} b^{i}(s) \partial_{i}^{+} \psi \varphi h^{d} \\
& -\sum_{i, j} \sum_{x} r^{2} \partial_{j}^{+} a^{i j}(s) \partial_{i}^{+} \psi \varphi h^{d}
\end{aligned}
$$




$$
\begin{gathered}
\quad+\sum_{x} r^{2} c(s) \psi \varphi h^{d} \mid \\
\leq K \sum_{i, j} \sum_{x} r^{2}\left|\rho^{2} \partial_{i}^{+} \psi \partial_{j}^{+} \varphi\right| h^{d} \\
+K \sum_{i} \sum_{x} r^{2}\left|\rho \partial_{i}^{+} \psi \varphi\right| h^{d}+K \sum_{x} r^{2}|\psi \varphi| h^{d} \\
\leq K \sum_{i}\left\|\rho \partial_{i}^{+} \psi\right\|_{l^{0,2}(r)} \sum_{j}\left\|\rho \partial_{j}^{+} \varphi\right\|_{l^{0,2}(r)} \\
+K \sum_{i}\left\|\rho \partial_{i}^{+} \psi\right\|_{l^{0,2}(r)}\|\varphi\|_{l^{0,2}(r)} \\
+K\|\psi\|_{l^{0,2}(r)}\|\varphi\|_{l^{0,2}(r)} \\
\leq K\|\psi\|_{l^{1,2}(r, \rho)} \cdot\|\varphi\|_{l^{1,2}(r, \rho)},
\end{gathered}
$$

where the same writing conventions are kept.

Owing to Theorem 3 the result follows.

\section{Approximation Results}

In this section, we study the approximation properties of scheme (17). We begin by investigating the consistency of the scheme and prove that the difference quotients approximate the partial derivatives (with accuracy of order 1). In addition, we estimate the rate of convergence of the difference quotients to the partial derivatives.

The result is obtained by using a Sobolev inequality, under stronger regularity assumptions, and imposing that the weights $\rho$ are bounded from below by a positive constant. In practice, the latter restriction amounts to assuming that the weights $\rho$ are increasing functions of $|x|$, which is precisely the case we are interested in.

Also, we note that the way we set our discrete framework, in strong connection with the framework for problem (7), plays a crucial role in obtaining the convergence rate.

Theorem 18. Let $r>0$ and $\rho>0$ be functions on $\mathbb{R}^{d}$ and $m$ an integer strictly greater than $d / 2$. Assume that (1)(2) in Assumption 5 are satisfied and also that $\rho(x) \geq C$ on $\mathbb{R}^{d}$, with $C>0$ being a constant. Let $u(t) \in W^{m+2,2}(r, \rho)$, $v(t) \in W^{m+3,2}(r, \rho)$, for all $t \in[0, T]$. Then there exists a constant $N$ independent of $h$ such that

(1) $\sum_{x \in Z_{h}^{d}} r^{2}(x)\left|u_{x^{i}}(t, x)-\partial_{i}^{+} u(t, x)\right|^{2} \rho^{2}(x) h^{d} \leq h^{2} N$ $\|u(t)\|_{W^{m+2,2}(r, \rho)}^{2}$,

(2) $\sum_{x \in Z_{h}^{d}} r^{2}(x)\left|v_{x^{i} x^{j}}(t, x)-\partial_{j}^{-} \partial_{i}^{+} v(t, x)\right|^{2} \rho^{4}(x) h^{d} \leq h^{2} N$ $\|v(t)\|_{W^{m+3,2}(r, \rho)}^{2}$

for all $t \in[0, T]$.

Remark 19. The following remarks will be used in the proof of the theorem.
(1) Under the conditions of the theorem, function $u(t)$ (function $v(t)$ ) has a modification in $x$ which is continuously differentiable in $x$ up to order 2 (up to order 3 ), and the derivatives equal the weak derivatives, for every $t \in[0, T]$. This can be proved by Sobolev's embedding of $W^{m, 2}(B)$ into $C^{n}(\bar{B})$, for balls $B$ in $\mathbb{R}^{d}$, if $m>d / 2+n$ (see, e.g., $[18,19]$ ). We consider these modifications in the theorem's proof.

(2) Note that if $U, V$ are open subsets of $\mathbb{R}^{d}$ with $V \subset U$ and $w \in W^{m, 2}(U)$ then $w \in W^{m, 2}(V)$. Also, if $w \in$ $W^{m, 2}(U)$ and $\zeta \in C_{0}^{\infty}(U)$ then $\zeta \in W^{m, 2}(U)$ and $\zeta w \in$ $W^{m, 2}(U)$ (see, e.g., $[18,19]$ ).

Proof of Theorem 18. Let us prove (1). We define a suitable geometric setting and then obtain an estimate for

$$
r^{2}(x)\left|u_{x^{i}}(t, x)-\partial_{i}^{+} u(t, x)\right|^{2} \rho^{2}(x),
$$

with $x \in Z_{h}^{d}$, using Sobolev's inequality on a fixed ball.

Let us consider $d$-cells

$$
\begin{gathered}
R_{h}=\left\{\left(x^{1}, x^{2}, \ldots, x^{d}\right) \in \mathbb{R}^{d}: x_{h}^{i}<x^{i}<x_{h}^{i}+h,\right. \\
i=1,2, \ldots, d\},
\end{gathered}
$$

with $x_{h}=\left(x_{h}^{1}, x_{h}^{2}, \ldots, x_{h}^{d}\right) \in Z_{h}^{d}$ fixed. Consider the particular $d$-cell, where $h=1$ and $x_{1}=(0,0, \ldots, 0)$, and denote it by $R_{1}^{0}$. Now, take open balls $B_{h}$ such that $B_{h} \supset R_{h}$, with the vertices $\left\{x_{h}^{i}, x_{h}^{i}+h, i=1,2, \ldots, d\right\}$ lying on the limiting sphere. Denote by $B_{1}^{0}$ the ball containing $R_{1}^{0}$.

For every $x_{h} \in Z_{h}^{d}$, taking in mind (1) in Remark 19, we have, by the mean-value theorem,

$$
\begin{aligned}
\partial_{i}^{+} u\left(t, x_{h}\right) & =h^{-1}\left(u\left(t, x_{h}+h e_{i}\right)-u\left(t, x_{h}\right)\right) \\
& =u_{x^{i}}\left(t, x_{h}+\theta h e_{i}\right)
\end{aligned}
$$

$$
\begin{aligned}
\left|u_{x^{i}}\left(t, x_{h}\right)-\partial_{i}^{+} u\left(t, x_{h}\right)\right| & =\left|u_{x^{i}}\left(t, x_{h}\right)-u_{x^{i}}\left(t, x_{h}+\theta h e_{i}\right)\right| \\
& \leq h\left|u_{x^{i} x^{i}}\left(t, x_{h}+\theta^{\prime} h e_{i}\right)\right|
\end{aligned}
$$

for some $0<\theta^{\prime}<\theta<1$. Clearly,

$$
\left|u_{x^{i} x^{i}}\left(t, x_{h}+\theta^{\prime} h e_{i}\right)\right| \leq \sup _{x \in R_{h}}\left|u_{x^{i} x^{i}}(t, x)\right|,
$$


and then, from (36) and (37),

$$
\left|u_{x^{i}}\left(t, x_{h}\right)-\partial_{i}^{+} u\left(t, x_{h}\right)\right|^{2} \leq h^{2} \sup _{x \in R_{h}}\left|u_{x^{i} x^{i}}(t, x)\right|^{2} .
$$

We change variable in order to have the supremum in (38) calculated over the fixed $d$-cell $R_{1}^{0}$ :

$$
\sup _{x \in R_{h}}\left|u_{x^{i} x^{i}}(t, x)\right|=\sup _{x \in R_{1}^{0}}\left|u_{x^{i} x^{i}}\left(t, x_{h}+h x\right)\right| \text {. }
$$

As

$$
\sup _{x \in R_{1}^{0}}\left|u_{x^{i} x^{i}}\left(t, x_{h}+h x\right)\right|^{2} \leq \sup _{x \in B_{1}^{0}}\left|u_{x^{i} x^{i}}\left(t, x_{h}+h x\right)\right|^{2},
$$

from (38)-(40) we immediately obtain

$$
\begin{aligned}
& r^{2}\left(x_{h}\right)\left|u_{x^{i}}\left(t, x_{h}\right)-\partial_{i}^{+} u\left(t, x_{h}\right)\right|^{2} \rho^{2}\left(x_{h}\right) \\
& \leq h^{2} \sup _{x \in R_{1}^{0}}\left(r^{2}\left(x_{h}+h x\right)\left|u_{x^{i} x^{i}}\left(t, x_{h}+h x\right)\right|^{2} \rho^{2}\left(x_{h}+h x\right)\right) \\
& \leq h^{2} \sup _{x \in B_{1}^{0}}\left(r^{2}\left(x_{h}+h x\right)\left|u_{x^{i} x^{i}}\left(t, x_{h}+h x\right)\right|^{2} \rho^{2}\left(x_{h}+h x\right)\right) .
\end{aligned}
$$

Now, taking in mind (2) in Remark 19, we have for $m>$ $d / 2$ by using Sobolev's inequality

$$
\begin{gathered}
\sup _{x \in B_{1}^{0}}\left|r\left(x_{h}+h x\right) u_{x^{i} x^{i}}\left(t, x_{h}+h x\right) \rho\left(x_{h}+h x\right)\right|^{2} \\
\leq N \sum_{|\alpha| \leq m} \int_{B_{1}^{0}} \mid D_{x}^{\alpha}\left(r\left(x_{h}+h x\right) u_{x^{i} x^{i}}\left(t, x_{h}+h x\right) \rho\right. \\
\left.\times\left(x_{h}+h x\right)\right)\left.\right|^{2} d x,
\end{gathered}
$$

with $N$ being a constant independent of $h$. Observe that the Leibniz formula

$$
\begin{aligned}
\left|D_{x}^{\alpha}\left(r u_{x^{i} x^{i}} \rho\right)\right| & =\left|\sum_{\beta \leq \alpha}\left(\begin{array}{l}
\alpha \\
\beta
\end{array}\right) D^{\beta}(r \rho) D_{x}^{\alpha-\beta} u_{x^{i} x^{i}}\right| \\
& =\left|\sum_{\beta \leq \alpha}\left(\begin{array}{l}
\alpha \\
\beta
\end{array}\right)\left(\sum_{\gamma \leq \beta}\left(\begin{array}{l}
\beta \\
\gamma
\end{array}\right) D^{\gamma} r D^{\beta-\gamma} \rho\right) D_{x}^{\alpha-\beta} u_{x^{i} x^{i}}\right|
\end{aligned}
$$

holds (the arguments of $r, \rho$ and $u_{x^{i} x^{i}}$ are omitted). Also, keeping the same convention, owing to Assumption 5

$$
\left|D^{\gamma} r\right| \leq K r \rho^{-|\gamma|}, \quad\left|D^{\beta-\gamma} \rho\right| \leq K \rho^{1-(|\beta|-|\gamma|)},
$$

with $K$ a constant, and then

$$
\begin{aligned}
\left|\sum_{\gamma \leq \beta}\left(\begin{array}{l}
\beta \\
\gamma
\end{array}\right) D^{\gamma} r D^{\beta-\gamma} \rho\right| & \leq N \sum_{\gamma \leq \beta}\left(\begin{array}{l}
\beta \\
\gamma
\end{array}\right) r \rho^{-|\gamma|} \rho^{1-(|\beta|-|\gamma|)} \\
& \leq N r \rho^{1-|\beta|},
\end{aligned}
$$

with $N$ a constant. From (42)-(45), we get

$$
\begin{array}{r}
\sup _{x \in B_{1}^{0}}\left|r\left(x_{h}+h x\right) u_{x^{i} x^{i}}\left(t, x_{h}+h x\right) \rho\left(x_{h}+h x\right)\right|^{2} \\
\leq N \sum_{|\alpha| \leq m} \sum_{\beta \leq \alpha} \int_{B_{1}^{0}} r^{2}\left(x_{h}+h x\right)\left|\rho^{1-|\beta|}\left(x_{h}+h x\right)\right|^{2} \\
\times\left|D_{x}^{\alpha-\beta} u_{x^{i} x^{i}}\left(t, x_{h}+h x\right)\right|^{2} d x .
\end{array}
$$

Note also that, owing to Hölder inequality and to the hypotheses on function $\rho$, the integral in (46) can be estimated by

$$
\begin{aligned}
& \int_{B_{1}^{0}} r^{2}\left(x_{h}+h x\right) \\
& \quad \times\left|\rho^{1-|\beta|}\left(x_{h}+h x\right) D_{x}^{\alpha-\beta} u_{x^{i} x^{i}}\left(t, x_{h}+h x\right)\right|^{2} d x \\
& \leq N \int_{B_{1}^{0}} r^{2}\left(x_{h}+h x\right) \mid \rho^{2+(|\alpha|-|\beta|)}\left(x_{h}+h x\right) \\
& \quad \times\left. D_{x}^{\alpha-\beta} u_{x^{i} x^{i}}\left(t, x_{h}+h x\right)\right|^{2} d x \\
& \quad \sup _{x \in B_{1}^{0}}\left|\rho^{-1-|\alpha|}\left(x_{h}+h x\right)\right|^{2} \\
& \leq N \int_{B_{1}^{0}} r^{2}\left(x_{h}+h x\right) \mid \rho^{2+(|\alpha|-|\beta|)}\left(x_{h}+h x\right) \\
& \times\left. D_{x}^{\alpha-\beta} u_{x^{i} x^{i}}\left(t, x_{h}+h x\right)\right|^{2} d x .
\end{aligned}
$$

Thus, from (46) and (47),

$$
\begin{gathered}
\sup _{x \in B_{1}^{0}}\left|r\left(x_{h}+h x\right) u_{x^{i} x^{i}}\left(t, x_{h}+h x\right) \rho\left(x_{h}+h x\right)\right|^{2} \\
\leq N \sum_{|\alpha| \leq m} \sum_{\beta \leq \alpha} \int_{B_{1}^{0}} r^{2}\left(x_{h}+h x\right) \\
\quad \times \mid \rho^{2+(|\alpha|-|\beta|)}\left(x_{h}+h x\right) \\
\left.\cdot D_{x}^{\alpha-\beta} u_{x^{i} x^{i}}\left(t, x_{h}+h x\right)\right|^{2} d x \\
\leq N \sum_{|\alpha| \leq m} \int_{B_{1}^{0}} r^{2}\left(x_{h}+h x\right) \\
\times\left|\rho^{2+|\alpha|}\left(x_{h}+h x\right) D_{x}^{\alpha} u_{x^{i} x^{i}}\left(t, x_{h}+h x\right)\right|^{2} d x
\end{gathered}
$$




$$
\begin{aligned}
& \leq N \sum_{|\alpha| \leq m+2} \int_{B_{1}^{0}} r^{2}\left(x_{h}+h x\right) \\
& \quad \times\left|\rho^{|\alpha|}\left(x_{h}+h x\right) D_{x}^{\alpha} u\left(t, x_{h}+h x\right)\right|^{2} d x \\
& =N \sum_{|\alpha| \leq m+2} \int_{B_{h}} r^{2}(x)\left|\rho^{|\alpha|}(x) D_{x}^{\alpha} u(t, x)\right|^{2} h^{-d} h^{2|\alpha|} d x \\
& \leq N \sum_{|\alpha| \leq m+2} \int_{B_{h}} r^{2}(x)\left|\rho^{|\alpha|}(x) D_{x}^{\alpha} u(t, x)\right|^{2} h^{-d} d x .
\end{aligned}
$$

Finally, owing to the particular geometry of the framework we have set, from (41) and (48) we obtain

$$
\begin{aligned}
& \sum_{x \in Z_{h}^{d}} r^{2}(x)\left|u_{x^{i}}(t, x)-\partial_{i}^{+} u(t, x)\right|^{2} \rho^{2}(x) h^{d} \\
& \quad \leq N h^{2} \sum_{|\alpha| \leq m+2} \sum_{x_{h} \in Z_{h}^{d}} \int_{B_{h}\left(x_{h}\right)} r^{2}(x)\left|\rho^{|\alpha|}(x) D_{x}^{\alpha} u(t, x)\right|^{2} d x \\
& \quad \leq N h^{2} \sum_{|\alpha| \leq m+2 x_{h} \in Z_{h}^{d}} \int_{R_{h}\left(x_{h}\right)} r^{2}(x)\left|\rho^{|\alpha|}(x) D_{x}^{\alpha} u(t, x)\right|^{2} d x \\
& \leq h^{2} N\|u(t)\|_{W^{m+2,2}(r, \rho)}^{2},
\end{aligned}
$$

where $B_{h}\left(x_{h}\right):=B_{h}, R_{h}\left(x_{h}\right):=R_{h}$, and the proof for (1) is complete.

The proof for (2) follows the same steps.

Finally, owing to the stability and consistency properties of the scheme (Theorems 17 and 18, resp.), we prove the convergence of the discrete problem's solution to the PDE problem's solution and compute a rate of convergence. The accuracy obtained is of order 1 .

Theorem 20. Let $r>0$ and $\rho>0$ be functions on $\mathbb{R}^{d}$ and $m$ an integer strictly greater than $d / 2$. Assume that the hypotheses of Theorems 11 and 17 are satisfied and that $\rho(x) \geq$ $C$ on $\mathbb{R}^{d}$, with $C>0$ being a constant. Denote by $u$ the solution of problem (7) in Theorem 11 and by $u_{h}$ the solution of problem (17) in Theorem 17. Assume additionally that $u \in$ $L^{2}\left([0, T] ; W^{m+3,2}(r, \rho)\right)$. Then

$$
\begin{aligned}
\sup _{0 \leq t \leq T}\left\|u(t)-u_{h}(t)\right\|_{l^{0,2}(r)}^{2}+\int_{0}^{T}\left\|u(t)-u_{h}(t)\right\|_{l^{1,2}(r, \rho)}^{2} d t \\
\leq h^{2} N \int_{0}^{T}\|u(t)\|_{W^{m+3,2}(r, \rho)}^{2} d t \\
\quad+N\left(\left\|g-g_{h}\right\|_{l^{0,2}(r)}^{2}+\int_{0}^{T}\left\|f(t)-f_{h}(t)\right\|_{l^{0,2}(r)}^{2} d t\right),
\end{aligned}
$$

with $N$ being a constant independent of $h$.

Remark 21. Under the conditions of the above theorem, there are modifications in $x$ such that the data $f(t)$ and $g$ are continuous in $x$, for every $t \in[0, T]$ (see Remark 19). We will consider these modifications in the proof of the theorem.

Proof of Theorem 20. From (7) and (17), we have that $u-u_{h}$ satisfies the problem

$$
\begin{gathered}
\left(u-u_{h}\right)_{t}=L_{h}\left(u-u_{h}\right)+\left(L-L_{h}\right) u+\left(f-f_{h}\right) \text { in } Q(h) \\
\left(u-u_{h}\right)(0, x)=\left(g-g_{h}\right)(x) \text { on } Z_{h}^{d} .
\end{gathered}
$$

Taking in mind Remark 21, we see clearly that $f-f_{h} \in$ $L^{2}\left([0, T] ; l^{0,2}(r)\right)$ and $g-g_{h} \in l^{0,2}(r)$.

With respect to the term $\left(L-L_{h}\right) u$, note that if $u(t) \in$ $W^{m+3,2}(r, \rho)$, for all $t \in[0, T]$,

$$
\begin{gathered}
\sum_{x \in Z_{h}^{d}} r^{2}(x)\left|\left(L-L_{h}\right)(t) u(t)\right|^{2} h^{d} \\
=\sum_{x \in Z_{h}^{d}} r^{2}(x) \mid a^{i j}(t, x)\left(\frac{\partial^{2}}{\partial x^{i} \partial x^{j}}-\partial_{j}^{-} \partial_{i}^{+}\right) u(t, x) \\
+\left.b^{i}(t, x)\left(\frac{\partial}{\partial x^{i}}-\partial_{i}^{+}\right) u(t, x)\right|^{2} h^{d} \\
\leq h^{2} N\|u(t)\|_{W^{m+3,2}(r, \rho)}^{2}<\infty,
\end{gathered}
$$

owing to (2) in Assumption 8 and to Theorem 18. Thus $\left(L-L_{h}\right)(t) u(t) \in l^{0,2}(r)$, for every $t \in[0, T]$. Moreover, as by assumption $u \in L^{2}\left([0, T] ; W^{m+3,2}(r, \rho)\right)$, we obtain immediately $\left(L-L_{h}\right) u \in L^{2}\left([0, T] ; l^{0,2}(r)\right)$.

We have shown that problem (51) satisfies the hypotheses of Theorem 17, therefore holding the estimate

$$
\begin{gathered}
\sup _{0 \leq t \leq T}\left\|u(t)-u_{h}(t)\right\|_{l^{0,2}(r)}^{2}+\int_{0}^{T}\left\|u(t)-u_{h}(t)\right\|_{l^{1,2}(r, \rho)}^{2} d t \\
\leq N\left(\left\|g-g_{h}\right\|_{l^{0,2}(r)}^{2}+\int_{0}^{T}\left\|f(t)-f_{h}(t)\right\|_{l^{0,2}(r)}^{2} d t\right. \\
\left.+\int_{0}^{T}\left\|\left(L-L_{h}\right)(t) u(t)\right\|_{l^{0,2}(r)}^{2} d t\right) .
\end{gathered}
$$

Owing again to (2) in Assumption 8 and to Theorem 18, the result follows.

The following result is an immediate consequence of Theorem 20.

Corollary 22. Let the hypotheses of Theorem 20 be satisfied, and denote by $u$ the solution of (7) in Theorem 11 and by $u_{h}$ the solution of (17) in Theorem 17. If there is a constant $N$ independent of $h$ such that

$$
\begin{aligned}
\| g & -g_{h}\left\|_{l^{0,2}(r)}^{2}+\int_{0}^{T}\right\| f(t)-f_{h}(t) \|_{l^{0,2}(r)}^{2} d t \\
& \leq h^{2} N\left(\|g\|_{W^{m, 2}(r, \rho)}^{2}+\int_{0}^{T}\|f(t)\|_{W^{m-1,2}(r, \rho)}^{2} d t\right)
\end{aligned}
$$


then

$$
\begin{gathered}
\sup _{0 \leq t \leq T}\left\|u(t)-u_{h}(t)\right\|_{l^{0,2}(r)}^{2}+\int_{0}^{T}\left\|u(t)-u_{h}(t)\right\|_{l^{1,2}(r, \rho)}^{2} d t \\
\leq h^{2} N\left(\int_{0}^{T}\|u(t)\|_{W^{m+3,2}(r, \rho)}^{2} d t+\|g\|_{W^{m, 2}(r, \rho)}^{2}\right. \\
\left.+\int_{0}^{T}\|f(t)\|_{W^{m-1,2}(r, \rho)}^{2} d t\right) .
\end{gathered}
$$

\section{Conclusions}

In this paper, we investigated the finite-difference spatial approximation of the Cauchy problem for a second-order linear parabolic PDE, in the framework of the variational approach.

By considering a suitable class of weighted Sobolev spaces, and its zero and first-order discrete versions, we could deal with the growth in space of the PDE coefficients (as well as with the spatial growth of the free data $f$ and $g$ ). Moreover, as the framework and techniques used to study the discrete problem mirror the framework and techniques for the corresponding continuous problem we could estimate a rate of convergence.

The approximation was studied under the strong assumption that the PDE does not degenerate. But the framework we used is broadly the appropriate framework for a future investigation of the related degenerate case.

Other possible further research directions include the use of splitting-up methods (see [10]), following Richardson's idea to accelerate numerical schemes, and also the use of techniques reducing the volume of computational work (e.g., sparse grid techniques), in order to deal with the computational challenge posed by the possible high dimensionality of the problem.

\section{Conflict of Interests}

The authors declare that there is no conflict of interests regarding the publication of this paper.

\section{Acknowledgments}

This research is supported by FCT, Fundação para a Ciência e Tecnologia (PEst-OE/EGE/UI0491/2011, Scientific Cooperation Agreement between Portugal and Slovakia-Analysis of Nonlinear Partial Differential Equations in Mathematical Finance), and by the European Commission, Marie Curie Multi-ITN STRIKE-Novel Methods in Computational Finance (Grant no. 304617).

\section{References}

[1] D. Lamberton and B. Lapeyre, Introduction to Stochastic Calculus Applied to Finance, Chapman \& Hall, London, UK, 1996.

[2] F. F. Gonçalves, Numerical approximation of partial differential equations arising in financial option pricing [Ph.D. thesis], University of Edinburgh, Edinburgh, UK, 2007.
[3] F. F. Gonçalves, M. D. R. Grossinho, and E. Morais, "Discretisation of abstract linear evolution equations of parabolic type," Advances in Difference Equations, vol. 2012, article 14, 29 pages, 2012.

[4] E. Morais, Analytical and numerical methods for some problems related to financial option pricing [Ph.D. thesis], University of Lisbon, Lisbon, Portugal, 2013.

[5] I. Gyöngy and N. V. Krylov, "Stochastic partial differential equations with unbounded coefficients and applications. I," Stochastics and Stochastics Reports, vol. 32, no. 1-2, pp. 53-91, 1990.

[6] V. Thomée, "Finite difference methods for linear parabolic equations," in Finite Difference Methods (Part 1)-Solution of Equations in $\mathbb{R}^{n}$, P. G. Ciarlet and J. L. Lions, Eds., vol. 1 of Handbook of Numerical Analysis, pp. 3-196, North-Holland, Amsterdam, The Netherlands, 1990.

[7] M. Broadie and J. B. Detemple, "Option pricing: valuation models and applications," Management Science, vol. 50, no. 9, pp. 1145-1177, 2004.

[8] I. Gyöngy and N. V. Krylov, "First derivatives estimates for finite-difference schemes," Mathematics of Computation, vol. 78, no. 268, pp. 2019-2046, 2009.

[9] I. Gyöngy and N. V. Krylov, "Higher order derivative estimates for finite-difference schemes for linear elliptic and parabolic equations," Methods and Applications of Analysis, vol. 16, no. 2, pp. 187-216, 2009.

[10] I. Gyöngy and N. V. Krylov, "Accelerated finite difference schemes for second order degenerate elliptic and parabolic problems in the whole space," Mathematics of Computation, vol. 80, no. 275, pp. 1431-1458, 2011.

[11] G. Barles, C. Daher, and M. Romano, "Convergence of numerical schemes for parabolic equations arising in finance theory," Mathematical Models \& Methods in Applied Sciences, vol. 5, no. 1, pp. 125-143, 1995.

[12] P. Lötstedt, J. Persson, L. von Sydow, and J. Tysk, "Spacetime adaptive finite difference method for European multi-asset options," Computers \& Mathematics with Applications, vol. 53, no. 8, pp. 1159-1180, 2007.

[13] D. Y. Tangman, A. Gopaul, and M. Bhuruth, "Numerical pricing of options using high-order compact finite difference schemes," Journal of Computational and Applied Mathematics, vol. 218, no. 2, pp. 270-280, 2008.

[14] D. M. Pooley, P. A. Forsyth, and K. R. Vetzal, "Numerical convergence properties of option pricing PDEs with uncertain volatility," IMA Journal of Numerical Analysis, vol. 23, no. 2, pp. 241-267, 2003.

[15] J. Douglas Jr. and T. F. Russell, "Numerical methods for convection-dominated diffusion problems based on combining the method of characteristics with finite element or finite difference procedures," SIAM Journal on Numerical Analysis, vol. 19 , no. 5, pp. 871-885, 1982.

[16] M. Ehrhardt, "Finite difference schemes on unbounded domains," in Advances in the Applications of Nonstandard Finite Difference Schemes, R. E. Mickens, Ed., vol. 2, pp. 343-384, World Scientific, River Edge, NJ, USA, 2005.

[17] M. Ehrhardt, "Discrete transparent boundary conditions for Schrödinger-type equations for non-compactly supported initial data," Applied Numerical Mathematics, vol. 58, no. 5, pp. 660-673, 2008. 
[18] J.-L. Lions and E. Magenes, Problèmes aux Limites non Homogènes et Applications, vol. 1, Dunod, Gauthier-Villars, Paris, France, 1968.

[19] L. C. Evans, Partial Differential Equations, vol. 19 of Graduate Studies in Mathematics, American Mathematical Society, Providence, RI, USA, 1998. 


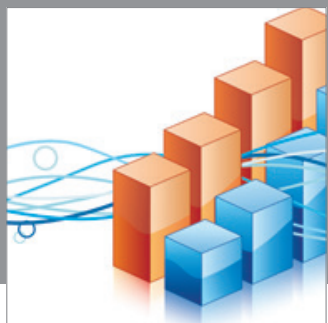

Advances in

Operations Research

mansans

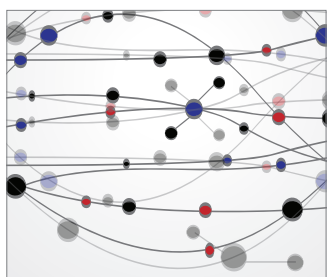

The Scientific World Journal
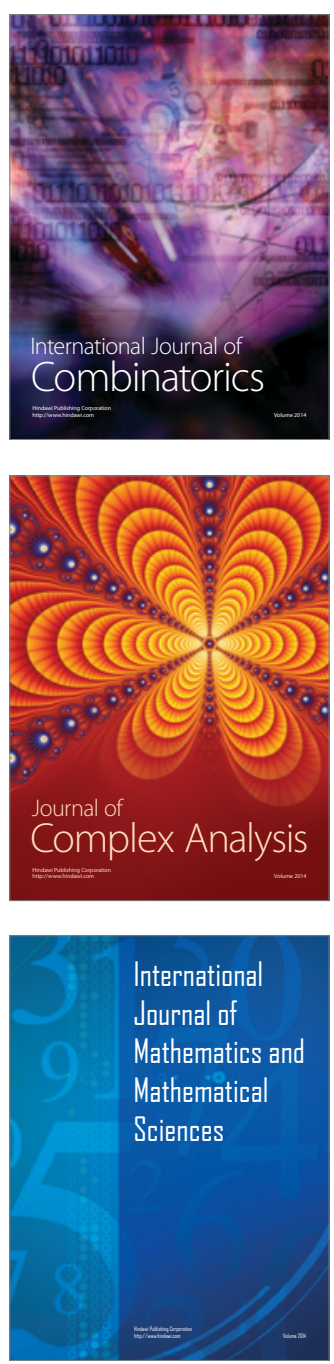
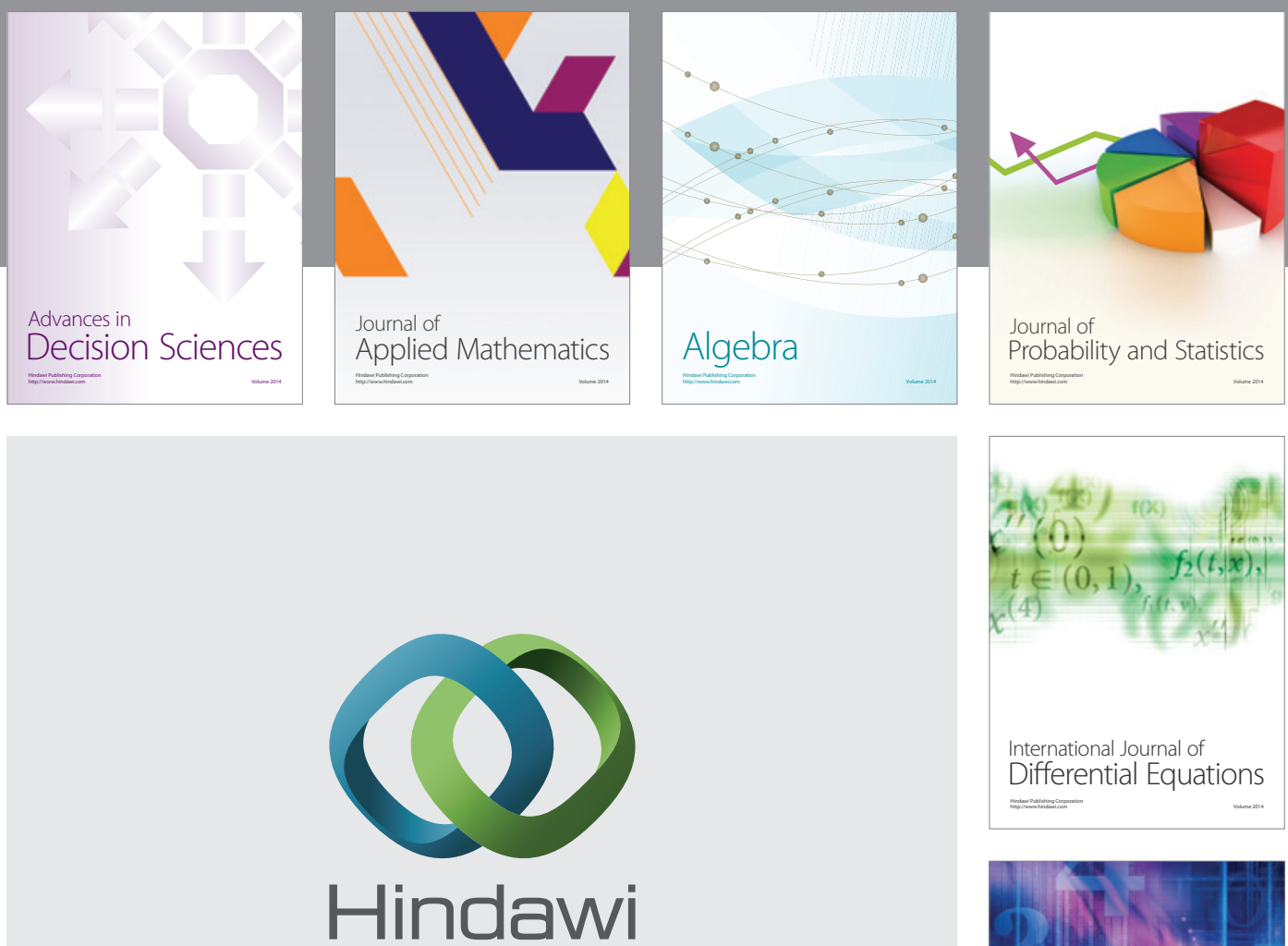

Submit your manuscripts at http://www.hindawi.com
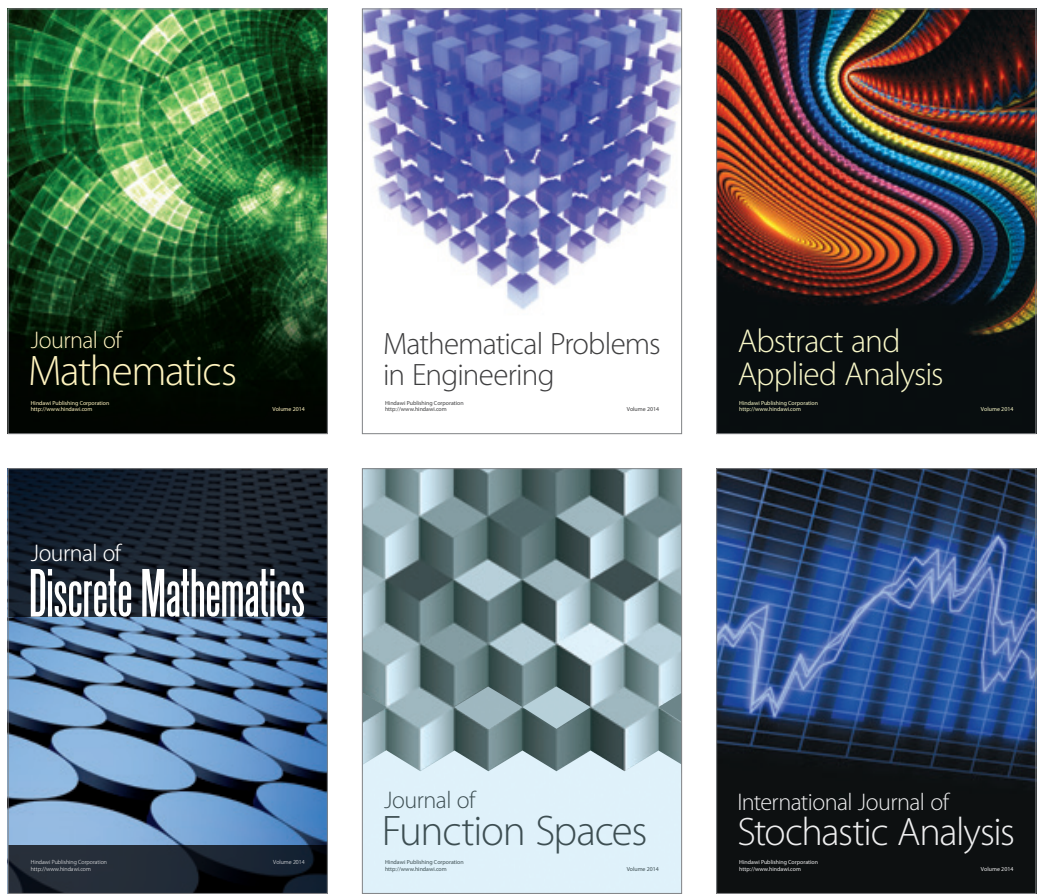

Journal of

Function Spaces

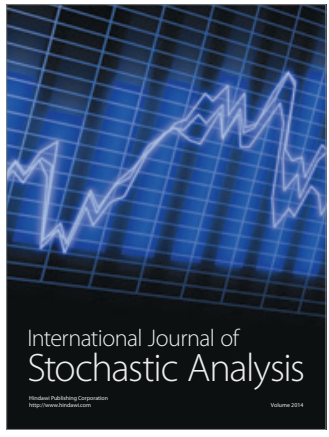

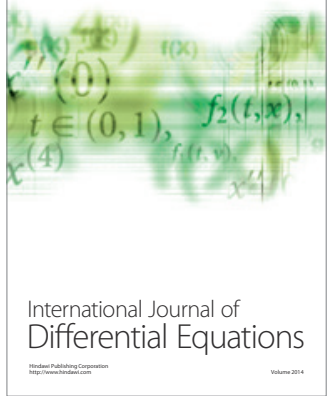
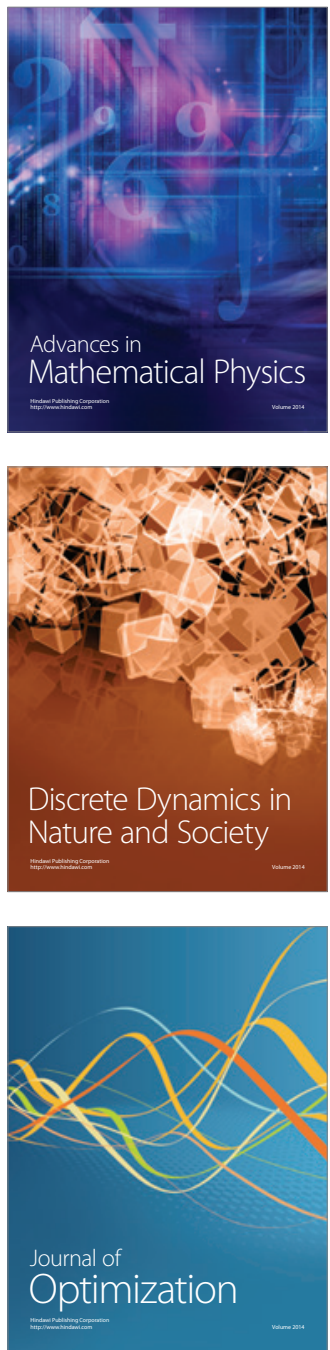